ELECTRONIC RESEARCH ANNOUNCEMENTS OF THE AMERICAN MATHEMATICAL SOCIETY

Volume 9, Pages 121-124 (November 26, 2003)

S $1079-6762(03) 00119-7$

\title{
SINGULARITY STRUCTURE IN MEAN CURVATURE FLOW OF MEAN-CONVEX SETS
}

\author{
TOBIAS H. COLDING AND BRUCE KLEINER
}

(Communicated by Svetlana Katok)

\begin{abstract}
In this note we announce results on the mean curvature flow of mean-convex sets in three dimensions. Loosely speaking, our results justify the naive picture of mean curvature flow where the only singularities are neck pinches, and components which collapse to asymptotically round spheres.
\end{abstract}

In this note we announce results on the mean curvature flow of mean-convex sets; all the statements below have natural generalizations to the setting of Riemannian 3 -manifolds, but for the sake of simplicity we will primarily discuss subsets of $\mathbb{R}^{3}$ here. Loosely speaking, our results justify the naive picture of mean curvature flow where the only singularities are neck pinches, and components which collapse to asymptotically round spheres. Recall that a one-parameter family of smooth hypersurfaces $\left\{M_{t}\right\} \subset \mathbb{R}^{n+1}$ flows by mean curvature if

$$
z_{t}=\mathbf{H}(z)=\Delta_{M_{t}} z,
$$

where $z=\left(z_{1}, \ldots, z_{n+1}\right)$ are coordinates on $\mathbb{R}^{n+1}$ and $\mathbf{H}=-H \mathbf{n}$ is the mean curvature vector. The papers ES91] and [CGG91] defined a level set flow for any closed subset $K$ of $\mathbb{R}^{n}$. This is a 1-parameter family of closed sets $K_{t} \subset \mathbb{R}^{n}$ with $K_{0}=K$ (if $K$ is a domain bounded by a smooth compact hypersurface, then the evolution of $\partial K$ for a short time interval coincides with the classical mean curvature evolution). Following [Whi00], we say that a compact subset $K \subset \mathbb{R}^{n}$ is mean-convex if $K_{t} \subset \operatorname{Int}(K)$ for all $t>0$. In this case there is also an associated Brakke flow $\mathcal{M}: t \mapsto M_{t}$ of rectifiable varifolds [Bra78, Ilm94, Whi00, and the pair $(\mathcal{M}, \mathcal{K})$, where

$$
\mathcal{K}:=\bigcup_{t \geq 0} K_{t} \times\{t\} \subset \mathbb{R}^{n} \times \mathbb{R}
$$

is called a mean-convex flow, Whi03. The fundamental papers Whi00, Whi03. developed a far-reaching partial regularity theory for mean curvature flow of meanconvex subsets of $\mathbb{R}^{n}$. Our results build on Whi00, Whi03, giving finer understanding of the singularities in the 3-dimensional case. Recall that the main result of [Whi00] asserts that the space-time singular set of the region swept out by a mean-convex set in $\mathbb{R}^{n+1}$ has parabolic Hausdorff dimension at most $(n-1)$, and

Received by the editors September 24, 2003.

2000 Mathematics Subject Classification. Primary 53C44.

Key words and phrases. Mean-convex, mean curvature, singularities.

The first author was supported by NSF grant DMS-0104453.

The second author was supported by NSF grant DMS-0204506.

(C)2003 American Mathematical Society 
Whi03 proved a structure theorem for blowups of mean-convex flows; cf. also HS99b, HS99a]. We expect that the more refined description of singularities given here will open the way for applications of mean-convex flow to geometric and/or topological problems involving mean-convex surfaces.

If $(\mathcal{M}, \mathcal{K})$ is a mean-convex flow in $\mathbb{R}^{3}$, then for almost every time $t$ the time slice $K_{t}$ is a domain with smooth boundary, Whi00, Corollary to Theorem 1.1]. Our first result shows that the high curvature portion of such smooth time slices has standard local geometry:

Theorem 1. For all $\epsilon>0$ there is a number $h_{0}=h_{0}(\epsilon)$ with the following property. If $(\mathcal{M}, \mathcal{K})$ is a mean-convex flow in $\mathbb{R}^{3}$ and $K_{t}$ is a regular time slice of $\mathcal{K}$ for some $t>0$, then there is a decomposition $K_{t}=G_{t} \cup B_{t}$ such that

- For all $x \in G_{t}$, and after rescaling by the factor $\frac{h_{0}}{d(x, \partial K)}$ the pointed subset $\left(K_{t}, x\right)$ is $\epsilon$-close to some pointed half-space $(P, p)$ in the pointed $C^{\frac{1}{\epsilon}}$ topology.

- Each component of $B_{t}$ is diffeomorphic to the 3-ball or a solid torus, and for all $x \in \partial K_{t} \cap B_{t}$, the pointed subset $\left(K_{t}, x\right)$ becomes, after rescaling by the factor $H(x), \epsilon$-close to a pointed convex model subset $(V, v)$ in the pointed $C^{\frac{1}{\epsilon}}$-topology. Here $V \subset \mathbb{R}^{3}$ is a convex set whose tangent cone at infinity is either a point, or a line, or a ray, and $V$ looks like a round cylinder near infinity, in the following sense: for every $\delta>0$ there is a compact set $K \subset V$ such that for every $v^{\prime} \in V$ lying outside $K$, if we rescale $V$ by $H\left(v^{\prime}\right)$, the resulting pointed subset $\left(V, v^{\prime}\right)$ is $\delta$-close to a round cylinder in the pointed $C^{\frac{1}{\delta}}$-topology.

Note that the bounds on the geometry deteriorate as one approaches $\partial K$; this is by necessity, since no regularity condition has been imposed on $K$. If $K$ happens to be smooth, then standard estimates for smooth mean curvature flow control the geometry of $K_{t}$ when $t \lesssim \sqrt{r}$, where $r$ is the normal injectivity radius of $\partial K$. Theorem 1 may be compared with the recent work of Huisken-Sinestrari [HS], in which a similar geometric description was obtained for mean curvature flow of smooth hypersurfaces in $\mathbb{R}^{n}$ where the sum of the first two principal curvatures is positive. The results in Per02, Sections 11, 12] are also in a similar spirit. Note that their results only apply to the evolution prior to the formation of the first singularity, whereas our results, like those in Whi00, Whi03], apply even after the formation of a singularity. (In fact, the methods yield a decomposition of arbitrary time slices, which we omit for the sake of simplicity.)

It follows from the strong maximum principle and compactness that the sets $\partial K_{t}$ for $t \geq 0$ are disjoint, and define a "singular foliation" of the original set $K$. Our next theorem proves Hölder regularity of the singular set of the foliation $\partial K_{t}$.

Theorem 2. The foliation defined by the sets $\partial K_{t}$ is smooth on the complement of a closed subset $S \subset K$ which satisfies the following Reifenberg-type condition: for all $\epsilon>0$ there is an $r_{0}=r_{0}(\epsilon)$ such that if $r<r_{0}$ and $x \in S$, then there is a line $A \subset \mathbb{R}^{3}$ such that $S \cap B(x, r)$ is contained in the tubular neighborhood $N_{\epsilon r}(A)$. In particular, $S$ lies in a 1-dimensional topological submanifold $\gamma \subset K$ which admits 
a $C^{\alpha}$-bi-Hölder parametrization for all $\alpha<1$. Furthermore, the mean curvature defines a proper function on $K \backslash S$.

After passing through a singularity the topological type of a surface flowing by mean curvature can change. In Whi95] White proved some results comparing the homology of the surface before and after such a singularity. Our next theorem shows that the region between two regular time slices is obtained from the earlier time slice by attaching 2 - and 3 -handles. Recall that attaching a $k$-handle to the boundary of an $n$-manifold $N$ is essentially just the process of attaching a fattenedup $k$-disk to $\partial N$ along the $(k-1)$-sphere, i.e. one glues $D^{k} \times D^{n-k}$ to $\partial N$ along $\partial D^{k} \times D^{n-k}$.

Theorem 3. If $0 \leq t<t^{\prime}$ and $K_{t}, K_{t^{\prime}}$ are regular time slices, then $K_{t} \backslash \operatorname{Int}\left(K_{t^{\prime}}\right)$ is a compact 3-manifold with boundary, which may be obtained from $\partial K_{t}$ by attaching $k$-handles for $k=2,3$.

Our final theorem deals with the mean-convex flow in a general 3-manifold, where the flow may converge as time tends to infinity to a set $K_{\infty}$ with nonempty interior.

Theorem 4. Let $M$ be a compact Riemannian 3-manifold, and $K \subset M$ a meanconvex subset with smooth boundary. Then as $t \rightarrow \infty$, the intersection of the sets $K_{t}$ converges to a (possibly empty) domain $K_{\infty} \subset \operatorname{Int}(K) \subset M$, where the boundary of $K_{\infty}$ is a smooth, weakly stable minimal surface, and $M \backslash \operatorname{Int}\left(K_{\infty}\right)$ may be obtained from $\partial K$ by attaching 2 - and 3 -handles. Furthermore, any compact minimal surface in $K \backslash \operatorname{Int}\left(K_{\infty}\right)$ is contained in $\partial K_{\infty}$; in particular $\partial K_{\infty}$ is homologically minimizing in the domain $K \backslash \operatorname{Int}\left(K_{\infty}\right)$.

The main novelty in this theorem is the assertion about the topology of $M \backslash \operatorname{Int}\left(K_{\infty}\right)$; the other statements can be deduced from earlier work.

\section{REFERENCES}

[Bra78] K. Brakke, The motion of a surface by its mean curvature, Mathematical Notes, vol. 20, Princeton University Press, Princeton, N.J., 1978. MR 82c:49035

[CGG91] Y. G. Chen, Y. Giga, and S. Goto, Uniqueness and existence of viscosity solutions of generalized mean curvature flow equations, J. Differential Geom. 33 (1991), no. 3, 749-786. MR 93a:35093

[ES91] L. C. Evans and J. Spruck, Motion of level sets by mean curvature. I, J. Differential Geom. 33 (1991), no. 3, 635-681. MR 92h:35097

[HS] G. Huisken and C. Sinestrari, in preparation.

[HS99a] Convexity estimates for mean curvature flow and singularities of mean-convex surfaces, Acta Math. 183 (1999), no. 1, 45-70. MR 2001c:53094

[HS99b] Mean curvature flow singularities for mean-convex surfaces, Calc. Var. Partial Differential Equations 8 (1999), no. 1, 1-14. MR 99m:58057

[Ilm94] T. Ilmanen, Elliptic regularization and partial regularity for motion by mean curvature, Mem. Amer. Math. Soc. 108 (1994), no. 520, x+90. MR 95d:49060

[Per02] G. Perelman, The entropy formula for the Ricci flow and its geometric applications, math.DG/0211159.

[Whi95] B. White, The topology of hypersurfaces moving by mean curvature, Comm. Anal. Geom. 3 (1995), no. 1-2, 317-333. MR 96k:58051 
[Whi00] - The size of the singular set in mean curvature flow of mean-convex sets, J. Amer. Math. Soc. 13 (2000), no. 3, 665-695. MR 2001j:53098

[Whi03] _ The nature of singularities in mean curvature flow of mean-convex sets, J. Amer. Math. Soc. 16 (2003), no. 1, 123-138. MR 2003g:53121

Courant Institute of Mathematical Sciences, 251 Mercer Street, New York, New YORK 10012

E-mail address: colding@cims.nyu.edu

Department of Mathematics, University of Michigan, 2072 East Hall, 525 E UniverSity Avenue, Ann Arbor, Michigan 48109-1109

E-mail address: bkleiner@umich.edu 